\section{KENTRON}

REVUE PLURIDISCIPLINAIR

DUMONDE ANTIQUE

\section{Kentron}

Revue pluridisciplinaire du monde antique

$31 \mid 2015$

Les Socratica de Xénophon

\title{
Il commentario inedito di Adolf Emper (ms. Leid. BPG 89, ff. 94r-103r) al Diogene o sulla tirannide (or. 6) di Dione di Prusa
}

\section{Gianluca Ventrella}

\section{(2) OpenEdition \\ Journals \\ Edizione digitale \\ URL: http://journals.openedition.org/kentron/381 \\ DOI: 10.4000/kentron.381 \\ ISSN: 2264-1459 \\ Editore \\ Presses universitaires de Caen}

\section{Edizione cartacea}

Data di pubblicazione: 1 novembre 2015

Paginazione: 165-184

ISBN: 978-2-84133-747-7

ISSN: 0765-0590

\section{Notizia bibliografica digitale}

Gianluca Ventrella, « Il commentario inedito di Adolf Emper (ms. Leid. BPG 89, ff. 94r-103r) al Diogene o sulla tirannide (or. 6) di Dione di Prusa », Kentron [En ligne], 31 | 2015, mis en ligne le 19 octobre 2016, consulté le 17 novembre 2020. URL : http://journals.openedition.org/kentron/381 ; DOI : https:// doi.org/10.4000/kentron.381

Questo documento è stato generato automaticamente il 17 novembre 2020.

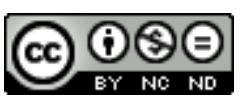

Kentron is licensed under a Creative Commons Attribution-NonCommercial-NoDerivatives 3.0 International License. 


\title{
Il commentario inedito di Adolf Emper (ms. Leid. BPG 89, ff. 94r-103r) al Diogene o sulla tirannide (or. 6) di Dione di Prusa
}

\author{
Gianluca Ventrella
}

1 Di recente riportato all'attenzione degli studiosi ${ }^{1}$, il Commentario alle orazioni dionee di Karl Wilhelm Emper (1807-1844) ${ }^{2}$ resta in grandissima parte ancora inedito ${ }^{3}$, nonostante l'interesse innegabile che lo stesso ha per la storia dell'ecdotica dionea. Scopo del presente contributo è quello di mettere a disposizione degli studiosi la sezione relativa all'or. 6 (Diogene o sulla tirannide) ${ }^{4}$, nell'auspicio, già espresso da Eugenio Amato, che si possa vedere, in un prossimo futuro, "l'edizione integrale di tale monumento » critico-esegetico ${ }^{5}$.

2 Prima di procedere alla trascrizione dell'inedito emperiano per la sezione relativa alla suddetta orazione, mi sia concesso soffermarmi brevemente sul modo di operare del filologo tedesco (§§ 1-2) e su un paio di passaggi del testo dioneo per il quale la lezione tràdita, contrariamente a quanto finora ritenuto dagli editori, potrebbe essere sana $\mathrm{e}$ non richiedere alcun intervento emendatorio $(\$ 3)$.

3 1. Va rilevato, innanzitutto, che Emper utilizzò il Commentario per correggere alcuni refusi ed errori materiali che ancora inquinavano la sua edizione degli opera omnia di Dione di Prusa. Tra questi il più emblematico è il seguente caso ${ }^{6}$ :

D.Chr., or. 6, 6:

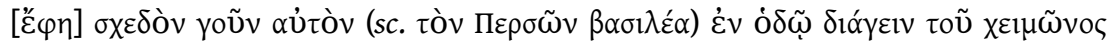

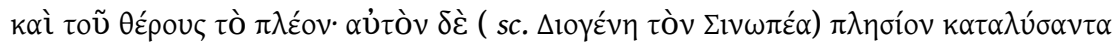

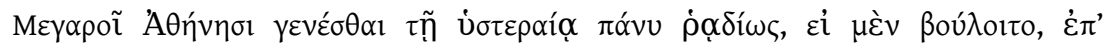

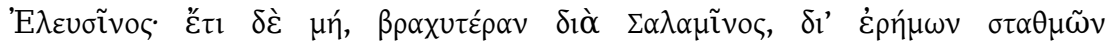

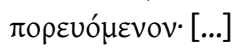

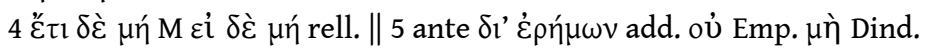




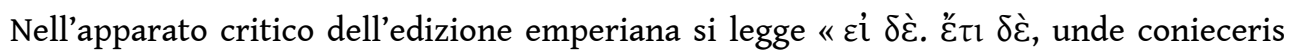

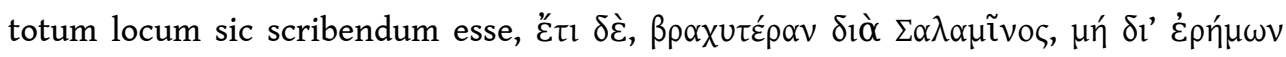

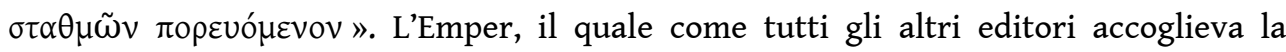
lezione $\varepsilon \dot{\imath} \delta \dot{\varepsilon}$, ometteva nella propria edizione l'indicazione del codice che trasmette la

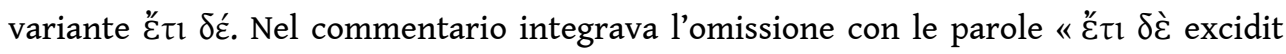
$\mathrm{M} »$. La dimenticanza può forse spiegare perché, degli editori successivi (i quali non ricollazionarono il manoscritto), nessuno abbia più ricordato tale lezione. Eppure, se preferito all' $\varepsilon i$ $\delta \varepsilon ́$ del resto della tradizione, il testo dell'autorevole Leidensis B.P.G. $2 \mathrm{C}$ (M) avrebbe resa superflua l'integrazione della negazione ov̉ davanti $\delta \imath^{\prime} \dot{\varepsilon} \rho \eta ́ \mu \omega \nu$ accettata, a partire dall'Emper in poi, da tutti gli editori. Si intenda infatti « diceva [...] che quello (sc. il Gran Re) trascorreva la maggior parte dell'inverno e dell'estate in cammino; lui (sc. Diogene di Sinope), invece, pernottando nei pressi di Megara, con estrema facilità raggiungeva dopo solo un giorno di viaggio Atene o, volendo, Eleusi ; perdippiù senza dover - accorciando per Salamina - far tappa in campi di sosta attraverso il deserto [...] ».

5 2. Il commentario costituì per l'Emper anche l'occasione per fornire una serie di loci similes $^{7}$ e talune utili indicazioni di lingua e stile ${ }^{8}$ fino ad allora mai indicate, ma anche, e soprattutto, l'opportunità per mettere a punto un approccio critico-esegetico più prudente. Lo studioso, infatti, mostra un atteggiamento generalmente più conservativo rispetto al textus receptus, sovente difeso tanto contro le ipotesi di emendazione dei suoi predecessori ${ }^{9}$, quanto contro talune sue proprie ipotesi testuali, alle quali egli dichiara ora di preferire la vulgata e, in particolare, il testo trasmesso dai codici ritenuti come più autorevoli ${ }^{10}$. Ad illustrare la maggiore attenzione mostrata dall'Emper nei confronti della lezione tràdita basti il seguente passaggio :

D.Chr., or. 6, 5 :

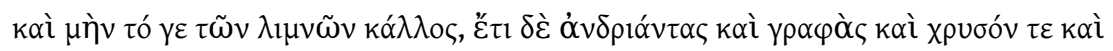

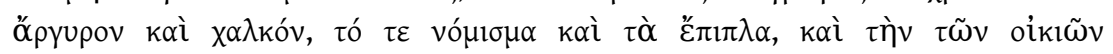

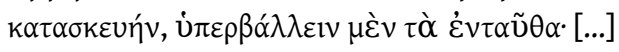

$\lambda_{1} \mu \nu \tilde{\omega} v$ codd. $\lambda_{\uparrow} \mu \varepsilon \dot{v} \omega \omega v$ omnes fere edd. post Cas.

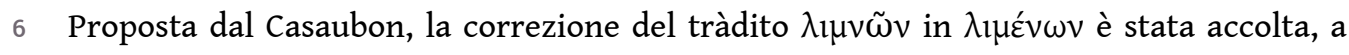
partire dal Morel, da tutti gli editori dionei (con la sola eccezione del Reiske ${ }^{11}$ e del Dukas $\left.^{12}\right)$, compreso, in un primo momento, lo stesso Emper. Questi, tuttavia, nel suo commentario ebbe a ricredersi, rilevando come la costruzione frastica e la logica del testo inducano a ritenere che le parole $\tau \grave{\alpha} \dot{\varepsilon} v \tau \alpha \tilde{v} \theta \alpha$ costituiscano l'oggetto dell'infinitiva, e non il suo soggetto, il quale deve riconoscersi, verosimilmente, nella

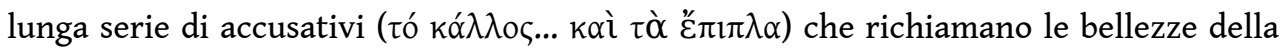
città di Babilonia messa a confronto con Atene. Si può pertanto rendere con: « $\mathrm{e}$ certamente almeno la bellezza dei laghi, e ancora le statue e i dipinti e le opere in oro, argento e bronzo, e la monetazione e l'arredamento e lo splendore delle case superano le cose di qui [sc. di Atene]». Siffatta interpretazione rende inutile l'emendazione proposta dal Casaubon, giacché la città di Babilonia, sita nell'entroterra assiro, non disponeva di porti ${ }^{13}$, mentre ben noti erano i laghi che ne costellavano il territorio ${ }^{14}$.

7 3. Forte dell'esempio emperiano, vorrei ora tentare di difendere, a mia volta, il testo tràdito contro $\mathrm{i}$ vari tentativi di emendazione accolti a testo dagli editori dionei, laddove invece la lezione tràdita potrebbe essere in realtà corretta.

D.Chr., or. 6, 2-3 :

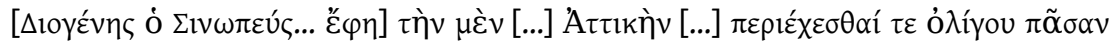




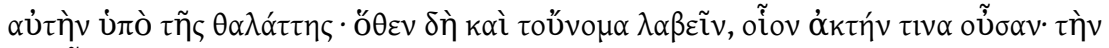

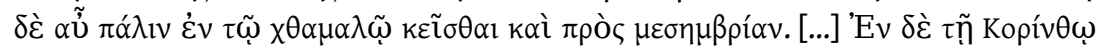

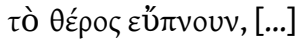
$\pi \alpha^{\prime} \lambda_{\imath v}$ codd. $\pi$ ó $\lambda_{\imath v}$ Rei. sed non necessario.

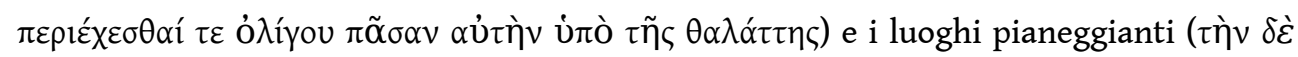

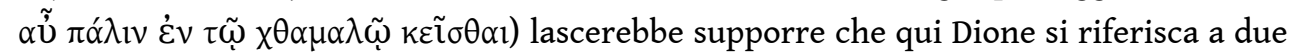
realtà distinte, nel primo caso alla regione (Attica), nel secondo alla città (Atene). Lo

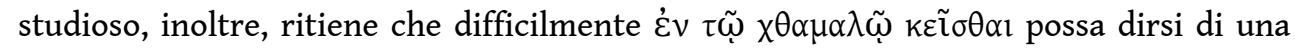

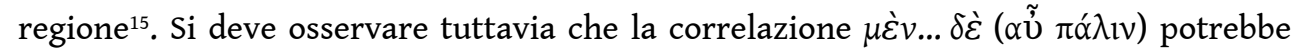
non esprimere una antitesi, ma avere un più generico valore connettivo ${ }^{16}$. In altri termini, non sarebbe necessario correggere, sull'esempio del Reiske (e di tutti gli

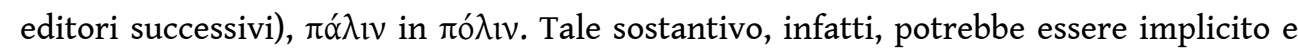
ricavabile dal contesto ${ }^{17}$ in cui si mettono a confronto le due città di Atene e di Corinto, la cui differente posizione geografica assicurava, rispettivamente, inverni miti ed estati fresche, ciò che consentiva a Diogene, cambiando la propria residenza in rapporto alle stagioni, di evitarne tutti i rigori. Si può pertanto interpretare : « Diogene di Sinope [...] diceva che l'Attica [...] è quasi tutta circondata dal mare : di qui prende il nome, come se fosse una akté; e quella (sc. città) poi è posta in pianura e rivolta a mezzogiorno. [...] A Corinto, invece, l'estate è ben ventilata [...] ».

D.Chr., or. 6, 36:

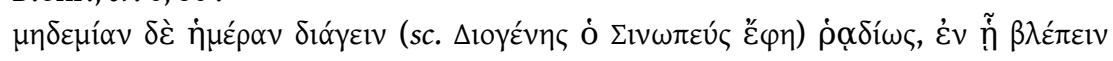

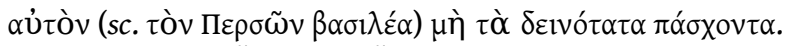

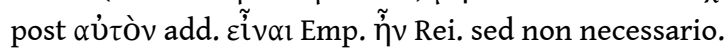

L'infinito è parso sospetto in quanto privo di un verbo reggente, che gli editori hanno variamente tentato di integrare ${ }^{18}$. Tuttavia si può sottintendere, sulla base del contesto, un verbo come ह̌ $\xi \varepsilon \sigma \tau \iota ~ o ~ \varepsilon ̌ \sigma \tau 1^{19}$. Del resto, non sarebbe questo l'unico caso in cui Dione sembra ricorrere ad una simile ellissi verbale ${ }^{20}$. Il senso, pertanto, sarà : «[sc. Diogene di Sinope diceva che il Re di Persia] nessun giorno trascorreva serenamente, senza (che fosse possibile) vederlo tra i peggiori tormenti ».

\section{BIBLIOGRAFIA}

AGUS A. (1999), Dione di Prusa. Diogene, o sulla tirannide (or. 6). Introduzione storico-tradizionale e criticotestuale. Edizione, traduzione e note critiche, Firenze, Università degli Studi (Dipartimento di Scienze dell'Antichità) (Tesi di Dottorato).

AMATO E. (2011), Xenophontis imitator fidelissimus. Studi su tradizione e fortuna erudite di Dione Crisostomo tra XVI e XIX secolo, Alessandria, Edizioni dell'Orso (Hellenica ; 40).

ARNIM H. von (1893), Dionis Prusaensis quem vocant Chrysostomum quae extant omnia. Edidit apparatu critico instruxit, vol. I, Berolini, Weidmann.

BOS L., SCHAEFER G.H. (1825), Lamberti Bos Ellipses Graecae, cum priorum editorum suisque observationibus edidit G.H. Schaefer, quibus adduntur Pleonasmi Graeci sive Commentarius de vocibus 
quae in sermone Graeco abundare dicuntur, auctore B. Weiske, una cum G. Hermanni dissertatione De ellipsi et pleonasmo in Graeca lingua, Londini, apud Ricardum Priestley.

BOULENGER F. (1922), Essai critique sur la syntaxe de l'Empereur Julien, Lille, Faculté catholique - Paris, Auguste Picard (Mémoires et travaux publiés par des professeurs des Facultés catholiques de Lille ; XXII).

BRUNCK R.F.P. (1812), Sophoclis Tragoediae septem, vol. I, Oxonii, e Typographeo Clarendoniano. BUDÉ G. de (1916), Dionis Chrysostomi Orationes, vol. I, Lipsiae, in aedibus B.G. Teubneri.

CASAUBON I. (1604), Diatriba, in Morel 1604 (paginazione autonoma).

DENNISTON J.D. (1950), The Greek Particles, Second Edition revised by K.J. Dover, Oxford, Oxford University Press, 1950 (rist. London, Gerald Duckworth \& Co. Ltd., 1996, 2002).

DINDORF L. (1857), Dionis Chrysostomi Orationes, I, Lipsiae, sumptibus et typis B.G. Teubneri.

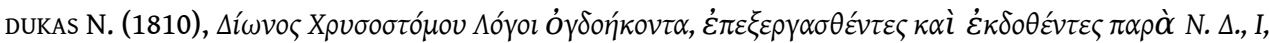

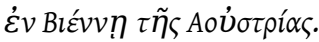

EMPER A. (1844), Dionis Chrysostomi Opera Graece, Brunsvigae, impensis G. Westermanni, 2 voll.

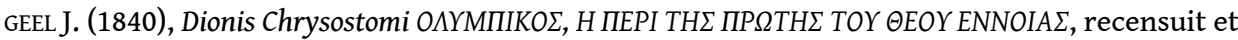
explicuit, commentarium de reliquis Dionis orationibus adjecit, Lugduni Batavorum, apud S. et J. Luchtmans.

GIANGRANDE G. (2002), « Sul testo della Oratio XI di Dione Crisostomo », Museum Philologum Londiniense, 11, p. 93-102.

KRAPINGER G. (1996), Dion Chrysostomos, Oratio 6, Text, Übersetzung und Kommentar, Graz, DBV-Verlag für die Technische Universität Graz (Dissertationen Karl-Franzens-Universität Graz ; 104).

KÜHNER R., GERTH B. (1898³), Ausführliche Grammatik der griechischen Sprache, 3 Aufl., II / 1-2, Satzlehre, Hannover, Hahnsche Buchhandlung.

MOREL F. (1604), Dionis Chrysostomi Orationes LXXX [...] Cum Is. Casauboni Diatriba, et eiusdem Morelli Scholiis, animadversionibus et coniectaneis [...], Lutetiae 1604 (rist. : 1623), ex Officina Typographica Claudii Morelli.

PFLUGK J. (1831), « Zu den Fragmenten des Euripides », Allgemeine Schulzeitung, 8, 2. Abth., Nr. 2-4, p. 9-30.

REISKE J.J. (1784), Dionis Chrysostomi Orationes, ex recensione I. I. R., cum eiusdem aliorumque animadversionibus [...], I, Lipsiae, ex Officina Sommeria, 1784.

VENTRELLA G. (2012), « Il commentario inedito di Adolf Emperius (ms. Leid. BPG 89, ff. 213r-267r) all' Olimpico di Dione di Prusa », GFA, 15, p. 1-60.

VENTRELLA G. (2015), « Da esercizio retorico a realtà vivente : la declamazione contro i tiranni nella polemica anti-domizianea di Dione di Prusa (or. 6) », in Présence de la déclamation antique : suasoires et controverses grecques et latines (Actes du colloque international de Clermont-Ferrand et Strasbourg, 17-18 novembre 2011 et 31 mai-1 ${ }^{\text {er }}$ juin 2012), R. Poignault, C. Schneider (dir.), 2015 (in c.d.s.).

\section{ALLEGATO}


Orat. V|21

De tyrannide

Oratio juvenilis et sententiis sophisticis referta (Geelius). Tyrannos molestissime vivere utpote a natura alienissimos. Quod declaratur tyrannorum et Diogenis vita comparata.

Diogenes Sinopensis gloriari solebat quod rex Persarum semet haud lautius viveret $(200,6)$. Quae ludibundus ille quidem dicebat, sed tamen ut ostenderet qualis esset ista quae dicitur felicitas. Nam bona ista partim inutilia esse, partim pauperrimo cuique prompta et parata $(200,16)$.

Neque enim, ut vulgo videbatur, aut vitae aut valetudinis incuriosior erat $(201,24)$. Imo vel jucunditatis rationem habebat, nimirum ejus quae e cultu victuque simplicissimo quaeritur. Sermonibus quoque commendare solebat et reprehendere si qui secus statuerent $(204,37)$. In primis vero sophistas irridebat qui ceteris se plus sapere opinarentur $(204,41)$. Docebat vero homines pejus vivere quam belluas, utpote minus secundum naturam; quas et valetudine meliore uti et vitam longius proferre $(205,17)$. Nec civitates ${ }^{[\mathrm{f} .92 \mathrm{v} .]}$ prodesse ad injuriam exterorum arcendam conditas ; nam ejusdem rei publicae cives gravissimis inter se injuriis afficere $(206,22)$. Male autem meritum de hominum genere Prometheum. Nam ignem luxus initium fuisse $(206,29)$. Deinde refellit hos qui corpus hominum negant ita natura comparatum esse ut belluarum modo vivant, sed ad voluptates abutantur $(207,19)$.

Quapropter quae nullo negotio comparari possent ad vitam sustentandam et aliqua jucunditate temperandam admittebat Diogenes ; luxuriam vero omnem excludebat $(208,35)$; semet quoque ajebat belluas imitari $(209,3)$. Ubi vero suam vitam cum ceterorum mortalium conditione comparabat, solum se videbat liberum et beatum esse $(210,19)$.

Ideo jam aspernabatur illam regis Persarum similitudinem. Et hunc enim et reliquos tyrannos (nam oratio Diogenis a rege Persarum paullatim ad solum genus tyrannorum delabitur) miserrimos esse $(210,21)$. Etenim sempiterno metu teneri omnique bonorum fructu privari, ut ${ }^{[\mathrm{f} .93 \mathrm{r} .]}$ tum demum recte videantur laetari cum trucidentur $(213,15)$. At dictu incredibile, tyrannos non sentire in maximis se malis versari, ut in isto cruciatu semper permaneant. Quo fieri ut et miserrime vivant et iidem mortem maxime formident $(214,34)$. Porro ut molestiae nullum eis finem esse, sic voluptatis nullum fructum ; ipsa enim perpetuitate ejus sensum hebescere $(214,42)$. Et divites tyranno suspectos esse et pauperes $(214,45)$. Neminem ei gratiam habere aliquo beneficio affectum $(214,3)$. Omnium invidiosissimum tyrannum esse $(214,5)$. Multa eum largiri opus esse, sed tamen suspectos esse eos quibus aliquid donaverit, et infensos hos qui, ut ista donaret, bonis exuendi fuerint $(215,12)$. Et propiora timet et remotiora $(215,16)$. Et pacem et bellum $(215,24)$. Et caritatem annonae et vilitatem $(215,26)$. Nihil omnino sine metu agit $(215,31)$. Omnium reliquorum tyrannorum caedes metuendo ipse perpetitur. Quo magis autem metuit, eo gnavius ei insidiantur $(216,45)$. Comparatur tyrannus cum homine inter gladios undecumque ei imminentes constituto $(216,5)$. Lenius agere Tantalum apud inferos $(216,10)$. Multis populis magnisque terris imperitantes miseriores esse quam unius civitatis et terrae exiguae dominos. Illis enim $^{[\text {f. } 93 \mathrm{v} .]}$ nullum refugium esse $(217,26)$. Suspecta est tyranno et libertas dicendi et ostentatio ; metuit generositatem pariter ac humilitatem secum conversantium (217, $33)$. Maledicta aegrius fert quam alii ; laude non delectatur $(217,36)$. Benevolentiae et amicitiae prorsus expers est $(217,42)$. 
Cum hac miserrima tyrannorum conditione jam suam comparat Diogenes, plane liberam a metu. Nam ne a terrae quidem motu vel lue vel fructuum calamitate quidquam sibi verendum esse.

\begin{tabular}{|c|c|}
\hline$\S 1,4^{[\mathrm{ff} .94 \mathrm{r} .]}$ & 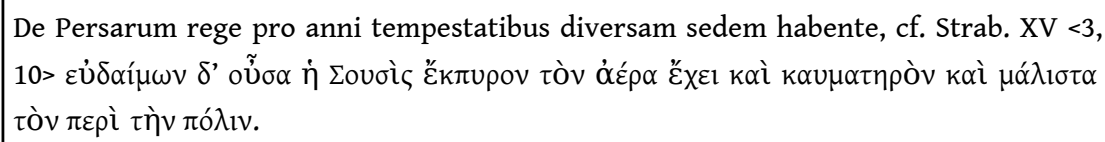 \\
\hline$\S 1,4$ & 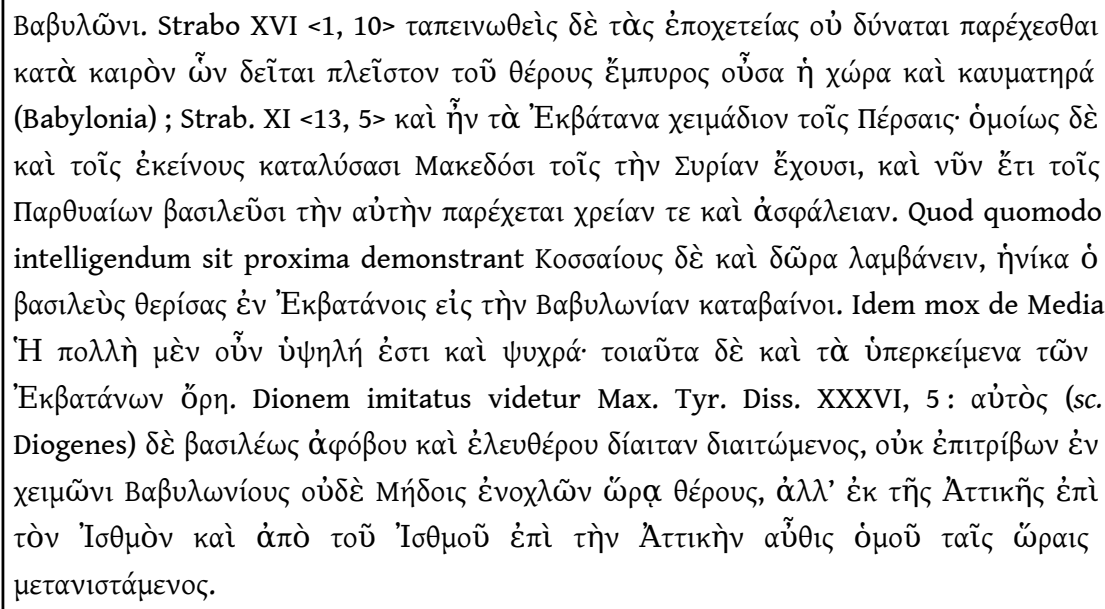 \\
\hline$\S 2,5$ & 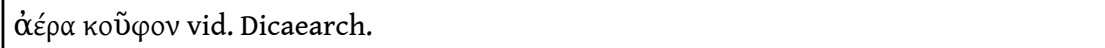 \\
\hline$\S 2,6$ & 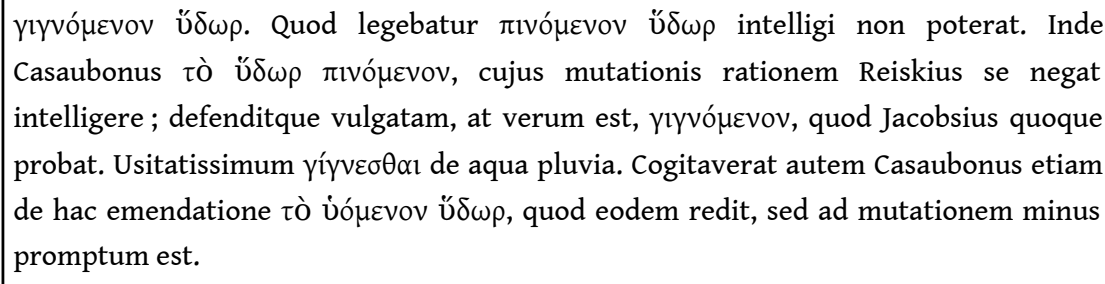 \\
\hline$\S 2,7$ & 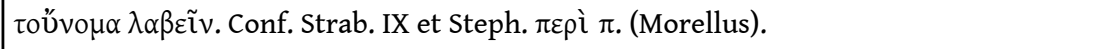 \\
\hline$\S 2,8^{[\mathrm{f.} 94 \mathrm{v}]}$ & 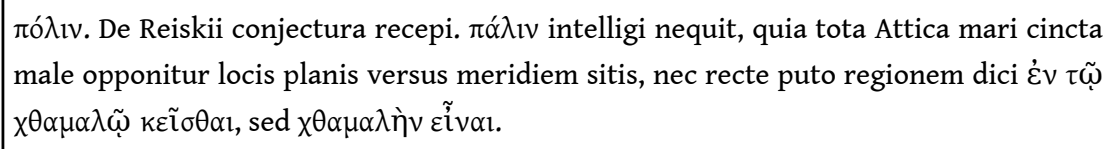 \\
\hline$\S 3,4$ & 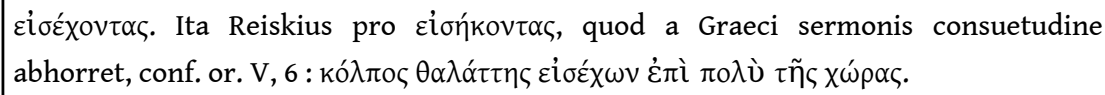 \\
\hline$\S 3,6$ & 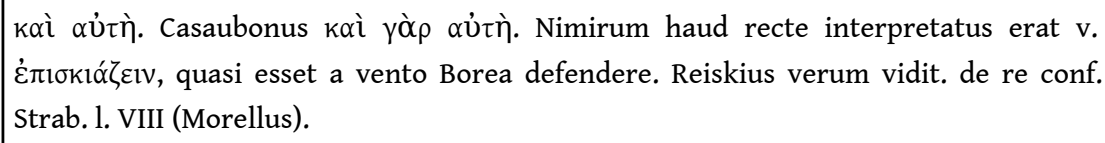 \\
\hline
\end{tabular}




\begin{tabular}{|c|c|}
\hline$\S 4,5$ & 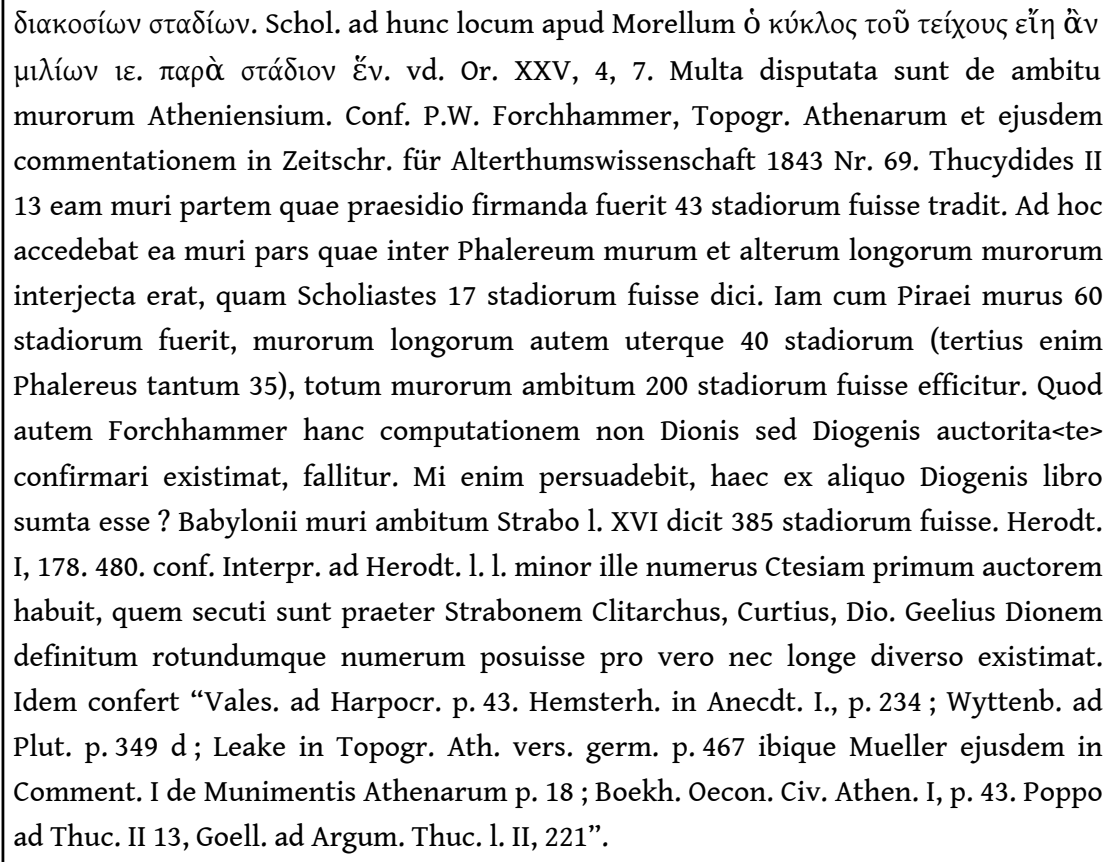 \\
\hline$\S 5,2$ & 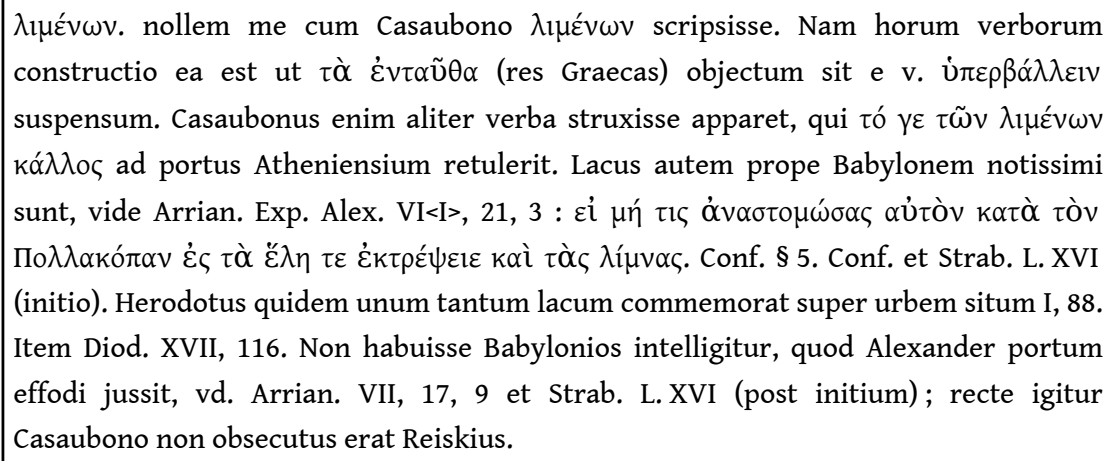 \\
\hline$\S 6,6$ & 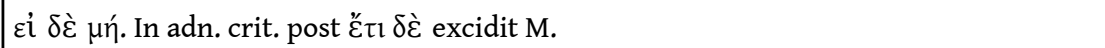 \\
\hline$\S 6,7$ & $\begin{array}{l}\text { oủ } \delta \mathrm{l}^{\prime} \dot{\varepsilon} \rho \eta ́ \mu \omega v . \text { Negationem reposui, qua oratio carere non poterat. Nam regem } \\
\text { quidem Persarum desertas stationes transire oportebat, non Diogenem Corintho } \\
\text { Athenas proficiscentem. }\end{array}$ \\
\hline$\S 8,2$ & 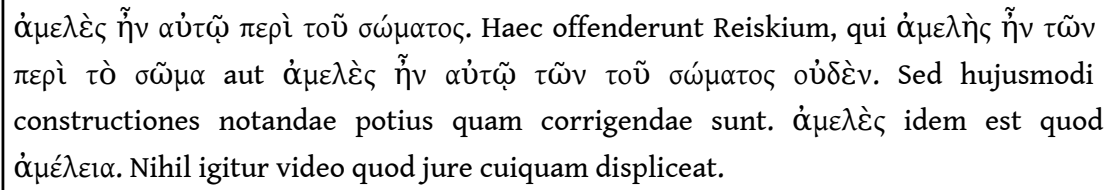 \\
\hline$\S 8,5$ & 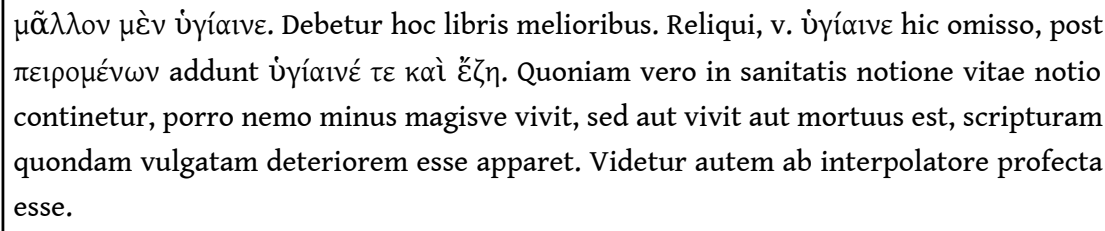 \\
\hline$\S 9,2$ & 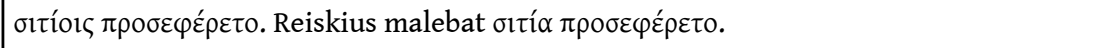 \\
\hline$\S 10,1$ & 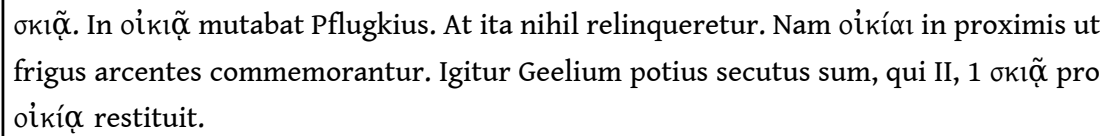 \\
\hline
\end{tabular}




\begin{tabular}{|c|c|}
\hline$\S 10,2$ & 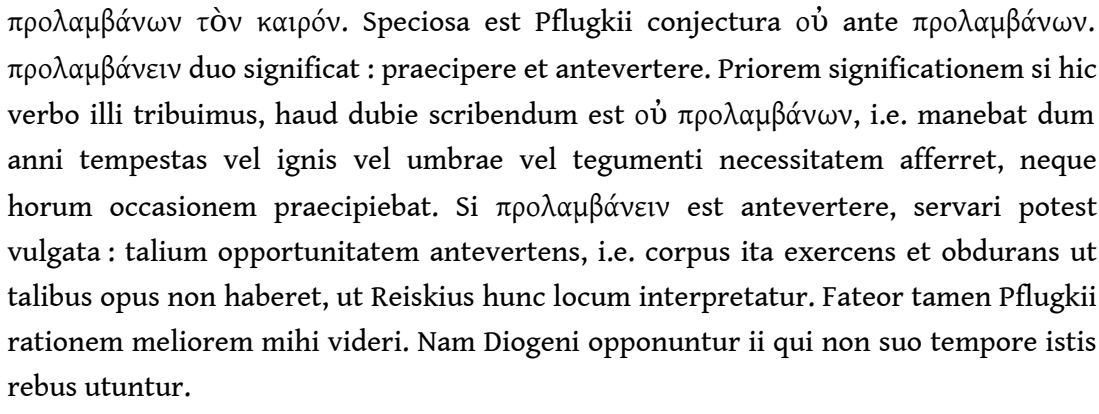 \\
\hline$\S 10,2$ & 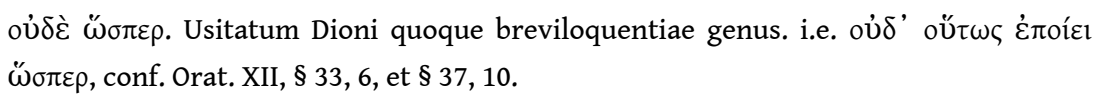 \\
\hline$\S 11,3$ & 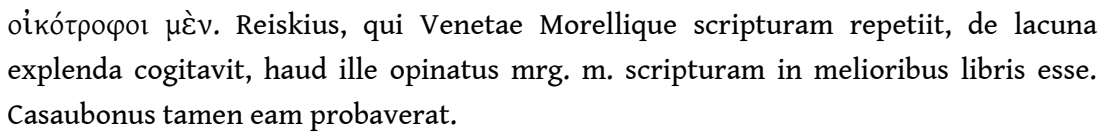 \\
\hline$\S 11,5$ & 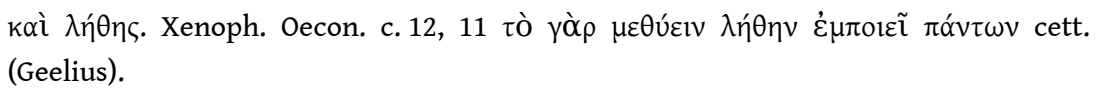 \\
\hline$\S 11,8$ & 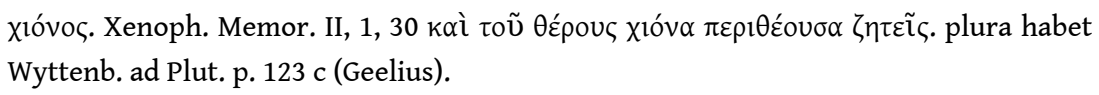 \\
\hline$\S 12,4$ & 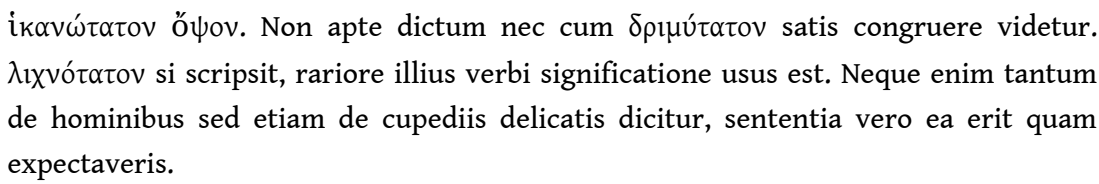 \\
\hline$\S 13,6^{[\mathrm{f} .97 \mathrm{v}]}$ & $\dot{\alpha} \pi \alpha \lambda \omega \tau \alpha \dot{\tau} \omega \nu$. Comparativus aptior est. Superlativus esset nimii fastidii. \\
\hline$\S 14,2$ & 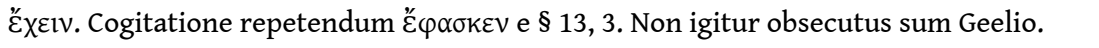 \\
\hline$\S 16,5$ & 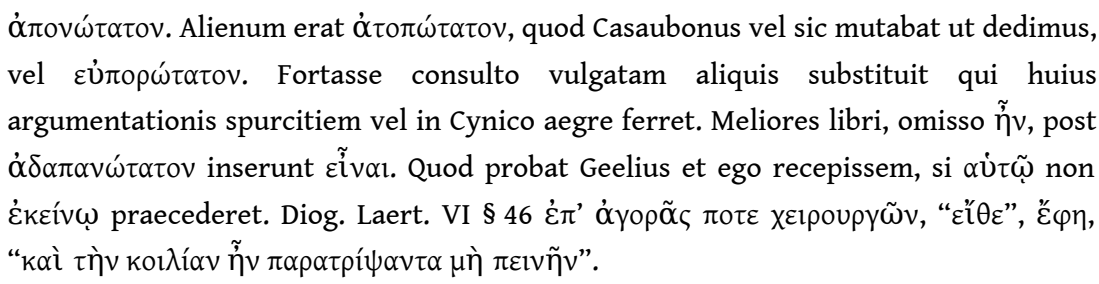 \\
\hline$\S 17,7$ & 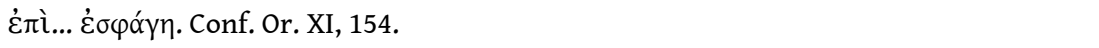 \\
\hline$\S 17,8$ & 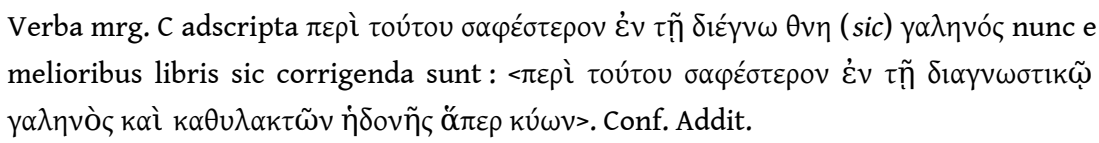 \\
\hline$\S 18,2$ & 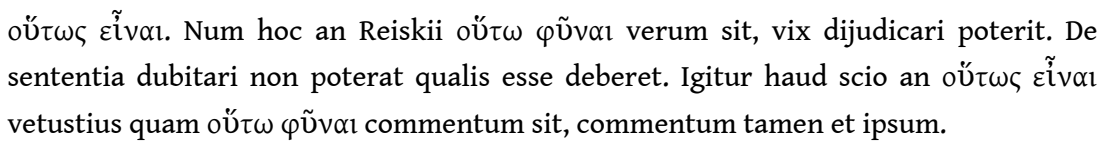 \\
\hline$\S 18,6^{[\mathrm{f} .98 \mathrm{r}]}$ & 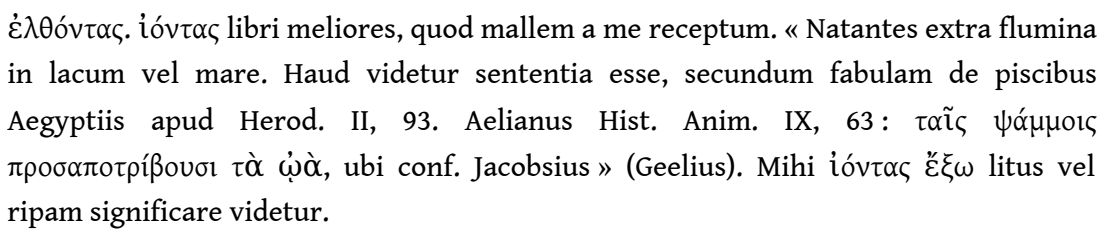 \\
\hline
\end{tabular}




\begin{tabular}{|c|c|}
\hline$\S 20,2$ & 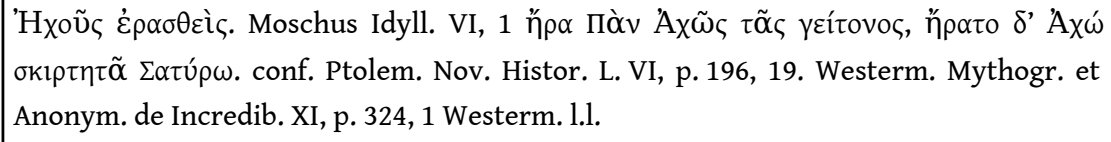 \\
\hline$\S 24$ & 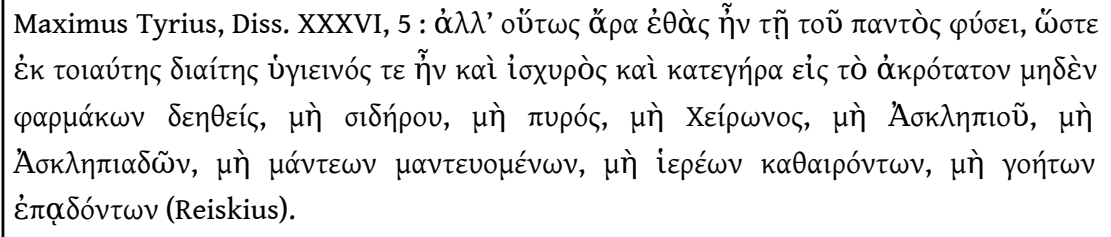 \\
\hline$\S 25,4^{[98 v]}$ & 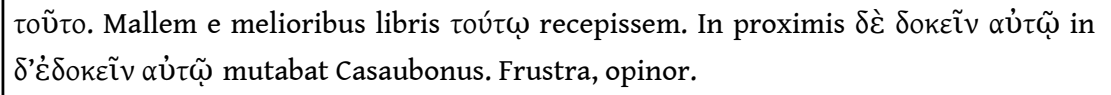 \\
\hline$\S 25,6$ & 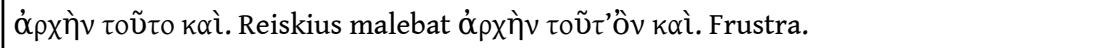 \\
\hline$\S 26,4$ & 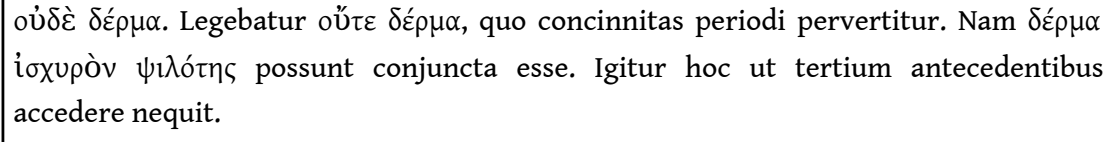 \\
\hline$\S 27,3$ & Post $\psi \tilde{u} \chi 0 \varsigma$ me invito comma pro colo legitur. \\
\hline$\S 28,6$ & 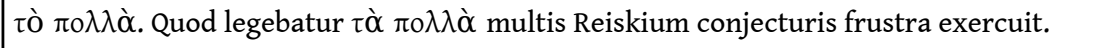 \\
\hline$\S 29,3$ & 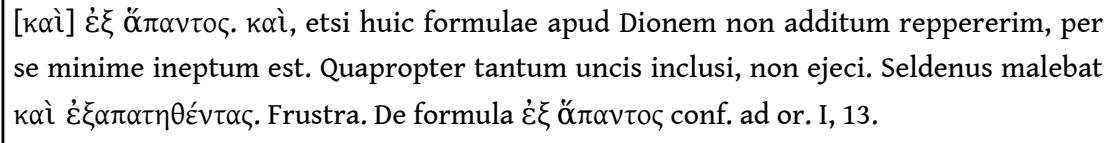 \\
\hline$\S 30,6$ & 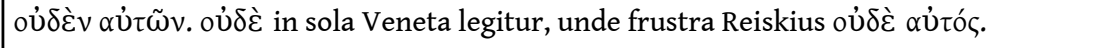 \\
\hline$\S 30,7$ & 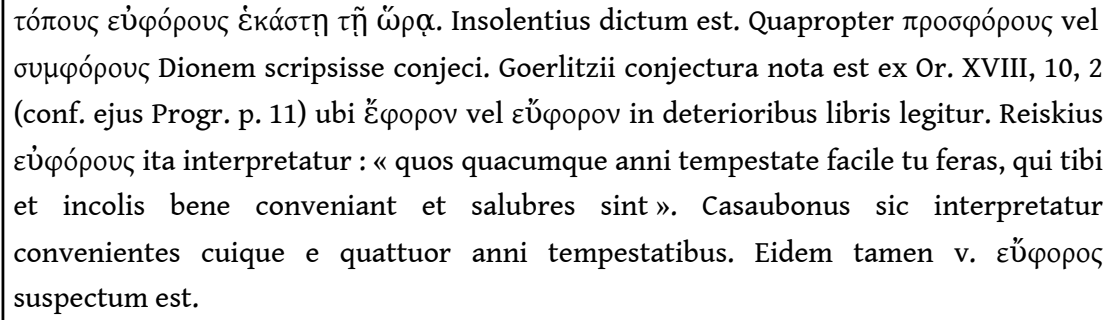 \\
\hline$\S 31,1$ & 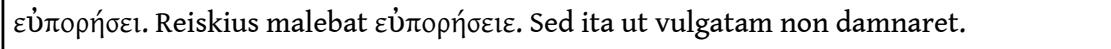 \\
\hline$\S 31,5$ & 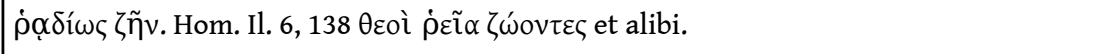 \\
\hline$\S 31,6$ & 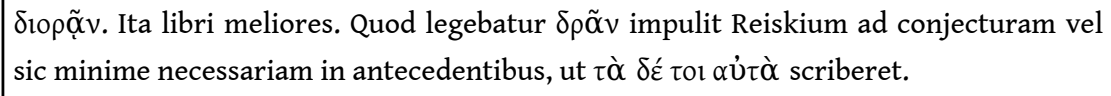 \\
\hline$\S 32,4$ & 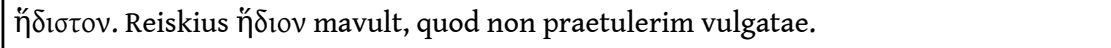 \\
\hline$\S 32,5$ & 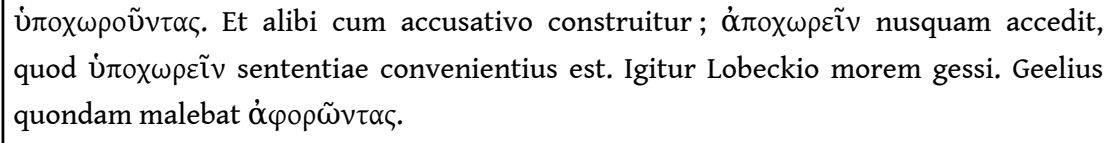 \\
\hline$\S 33,2$ & 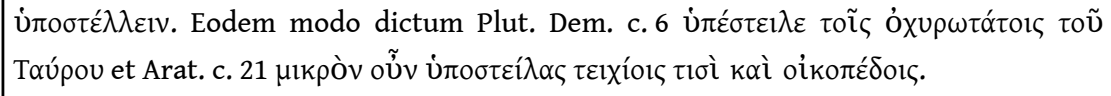 \\
\hline$\S 33,4$ & 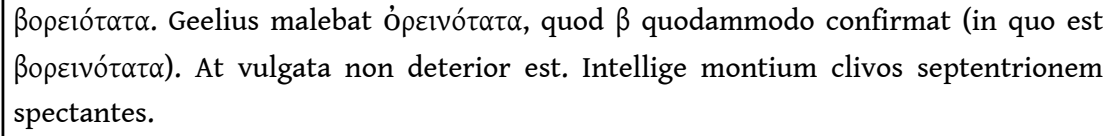 \\
\hline$\S 34,3$ & 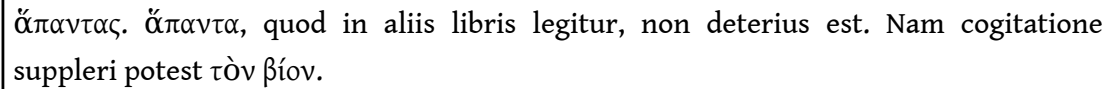 \\
\hline
\end{tabular}




\begin{tabular}{|c|c|}
\hline$\S 34,7$ & 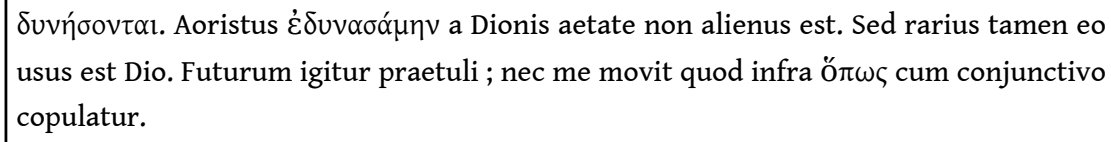 \\
\hline$\S 34,12^{[100 \mathrm{r}]}$ & 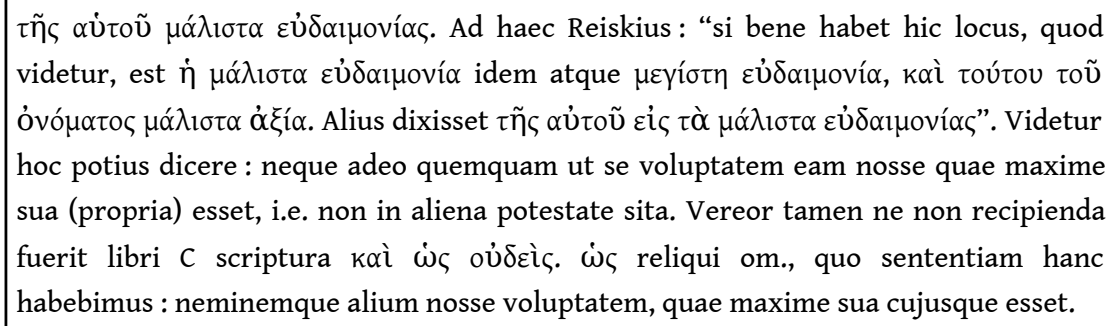 \\
\hline$\S 36,4$ & 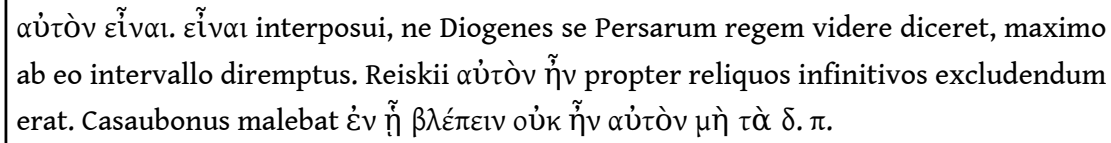 \\
\hline$\S 37,4$ & 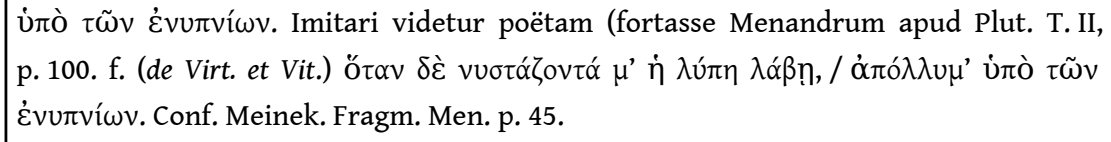 \\
\hline$\S 37,4^{[\mathrm{f} .100 \mathrm{v}]}$ & 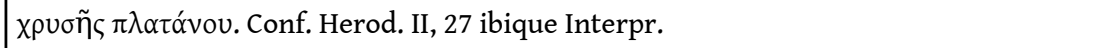 \\
\hline$\S 40,7$ & 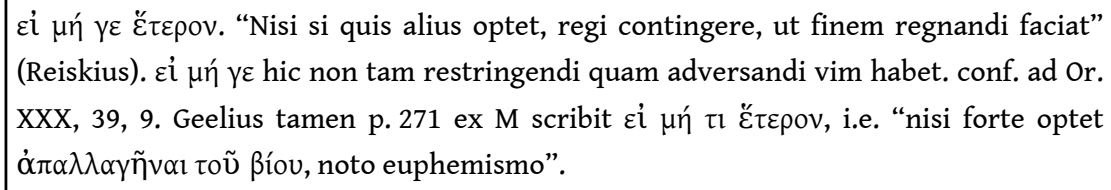 \\
\hline$\S 40,10$ & 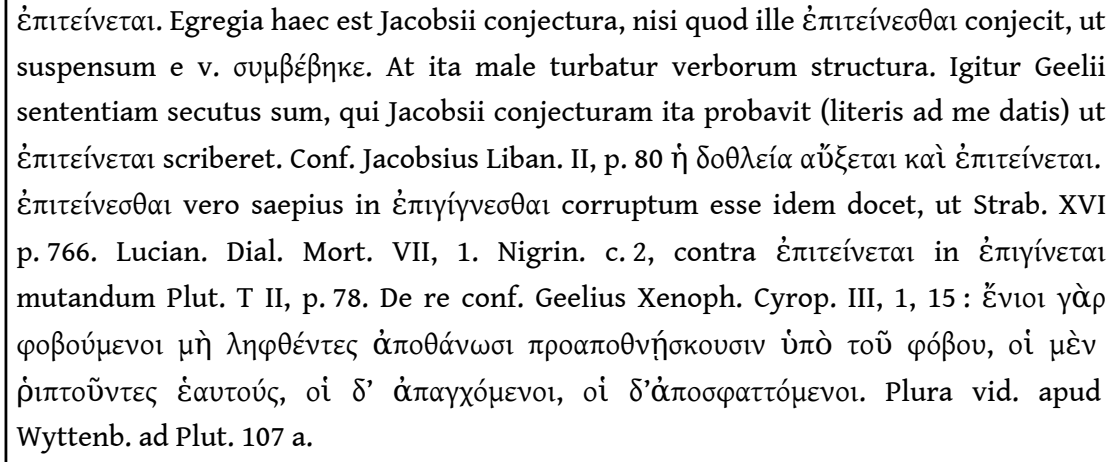 \\
\hline$\S 41,2^{[f .101 r]}$ & 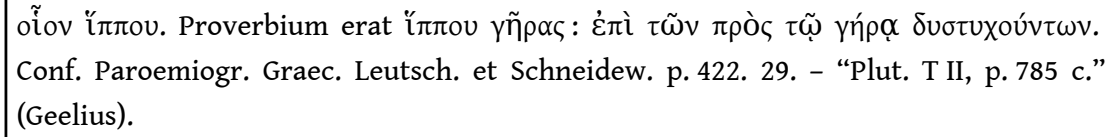 \\
\hline$\S 42,3$ & 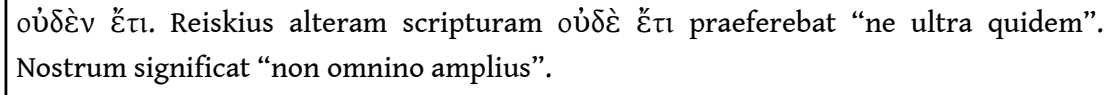 \\
\hline$\S 42,7$ & $\begin{array}{l}\pi \varepsilon \rho ı \varepsilon \imath \eta \varphi o ́ \tau \omega v . \text { Hoc conjeceram postulante sententia "hostibus undique circumfusis" } \\
\text { eamque conjecturam eatenus confirmarunt libri meliores ut } \pi \alpha \rho \varepsilon \imath \lambda \eta \varphi o ́ \tau \omega v \text { habent. } \\
\text { Vulgo enim } \pi \rho \circ \varepsilon \imath \lambda \eta \varphi ́ \tau \omega v \text { legebatur. }\end{array}$ \\
\hline
\end{tabular}




\begin{tabular}{|c|c|}
\hline$\S 43,6$ & 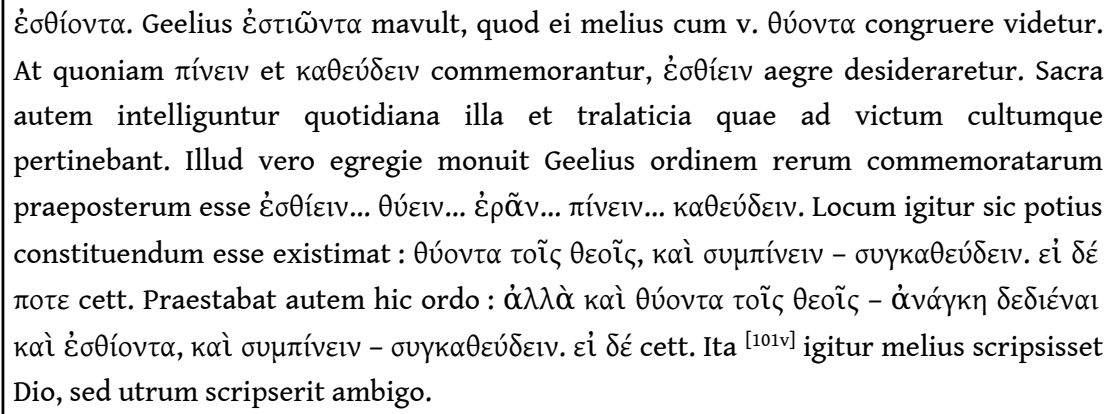 \\
\hline$\S 44,2$ & 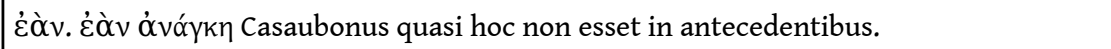 \\
\hline$\S 44,4$ & 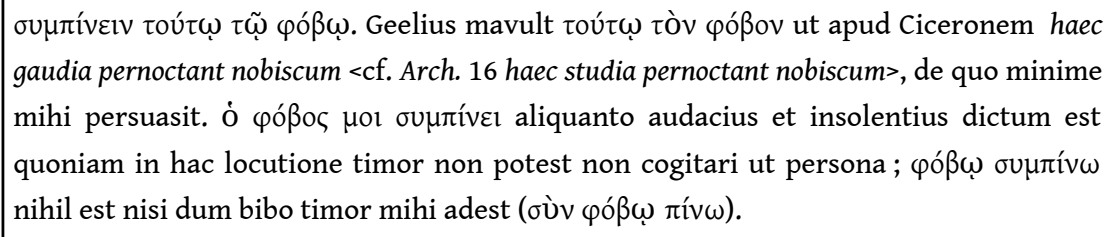 \\
\hline$\S 44,5$ & $\begin{array}{l}\mu o ́ v o v \text { primus proposuit Jacobsius. Quod in } C \mu \varepsilon ̀ v \text { (quae vulgata scriptura erat) deest, } \\
\text { correctoris industriam indicat. Reiskius vel delebat vel in } \mu \varepsilon ́ \gamma \alpha \text { mutabat. }\end{array}$ \\
\hline$\S 45,4$ & 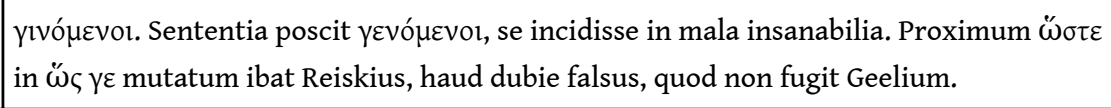 \\
\hline$\S 45,5^{[102 r]}$ & 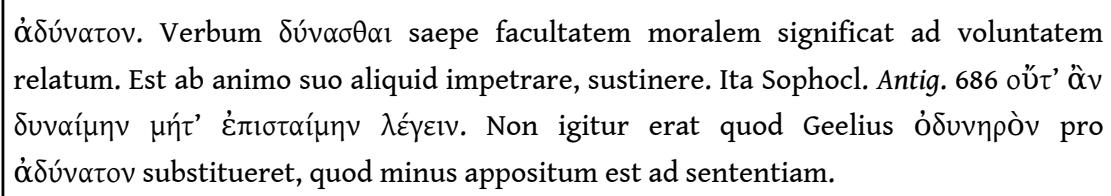 \\
\hline$\S 45,8$ & 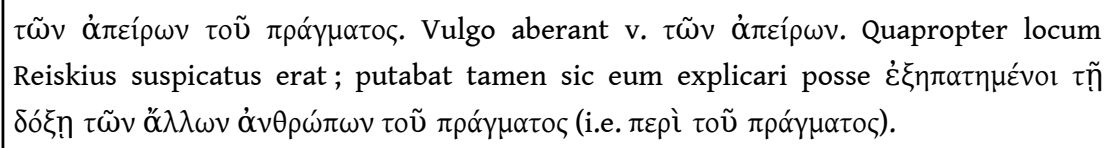 \\
\hline$\S 47,2$ & 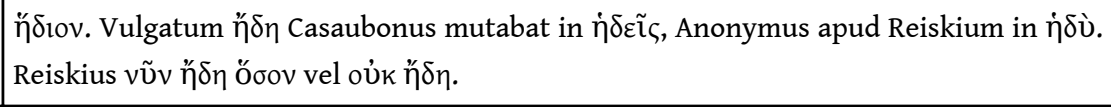 \\
\hline$\S 47,3$ & 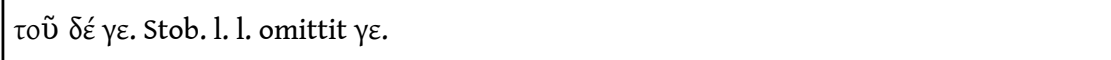 \\
\hline$\S 50,9$ & 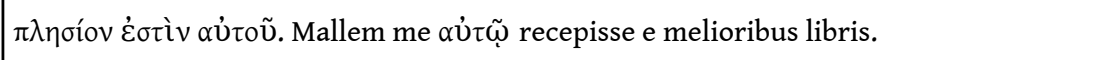 \\
\hline$\S 52,4$ & 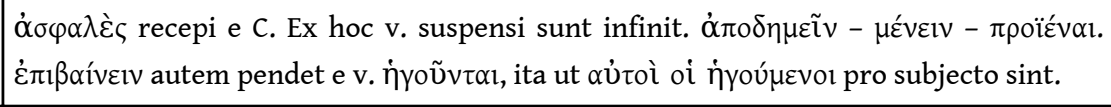 \\
\hline$\S 53,2$ & ǒ $\sigma \alpha 1$. Reiskius, quod in Veneta ǒ $\sigma \alpha$ esse videret, ǒ $\sigma \omega v$ malebat. \\
\hline$\S 53,5$ & 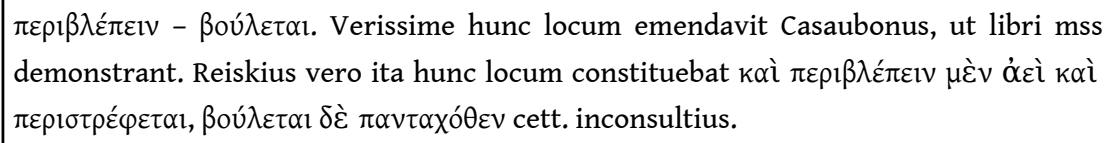 \\
\hline$\S 54,3$ & 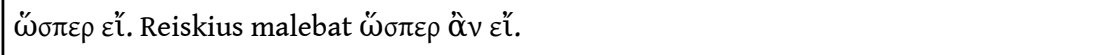 \\
\hline$\S 54,4^{[\mathrm{f} .103 \mathrm{r}]}$ & 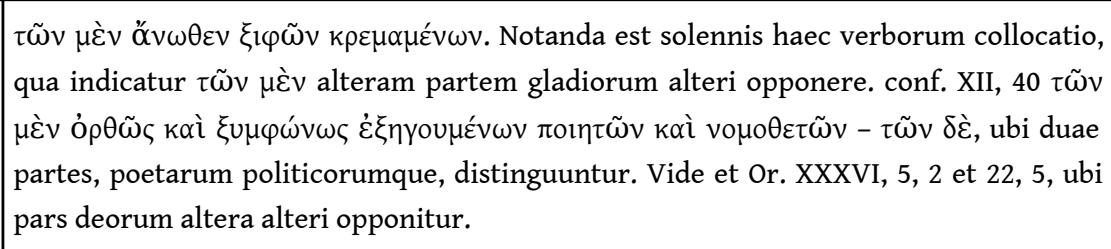 \\
\hline
\end{tabular}




\begin{tabular}{|c|c|}
\hline$\S 55,4$ & 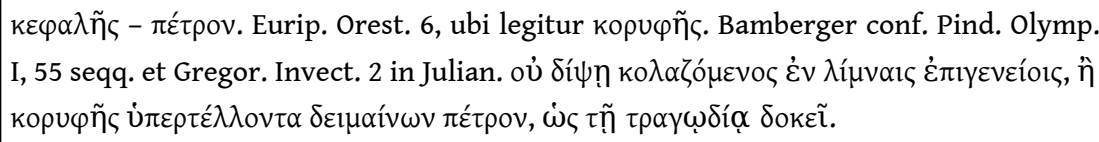 \\
\hline$\S 56,2$ & 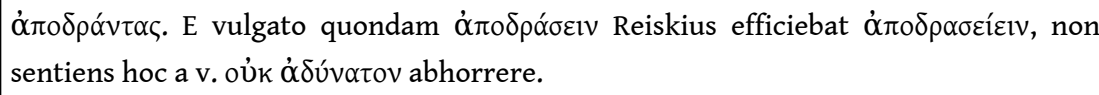 \\
\hline$\S 57,1$ & 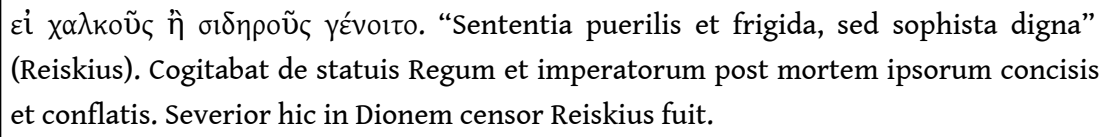 \\
\hline$\S 58,1$ & $\dot{\varepsilon} \lambda \varepsilon v \theta \varepsilon p i ́ \omega \varsigma$ est etiam in A. \\
\hline$\S 59,2$ & 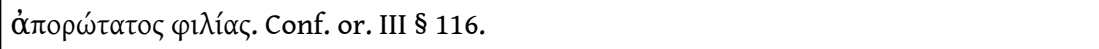 \\
\hline$\S 59,3$ & 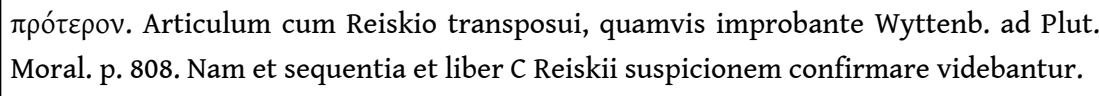 \\
\hline$\S 61,2$ & 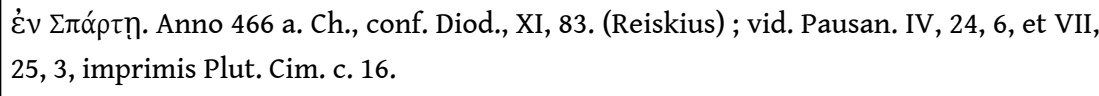 \\
\hline$\S 62,4$ & 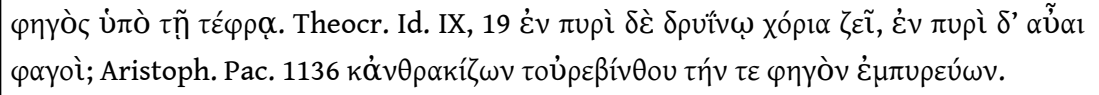 \\
\hline$\S 62,4$ & 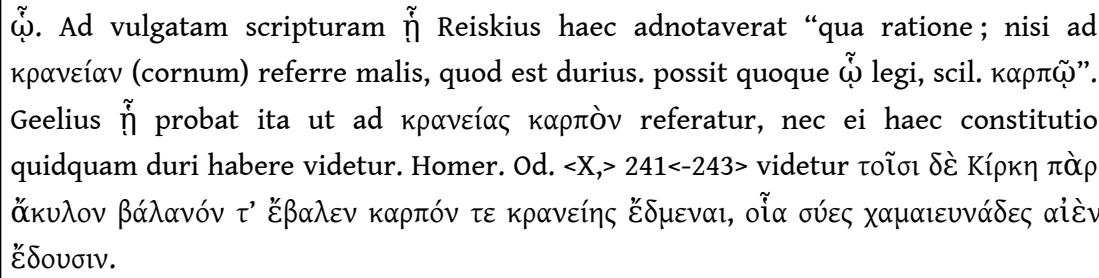 \\
\hline
\end{tabular}

\section{Lista delle abbreviazioni bibliografiche usate da Emper}

\begin{tabular}{|l|l|}
\hline Addit. & Emper 1844, p. 806-807. \\
\hline $\begin{array}{l}\text { Boekh. Oecon. Civ. } \\
\text { Athen. }\end{array}$ & $\begin{array}{l}\text { Böckh A. (1817), Die Staatshaushaltung der Athener, Berlin, in der } \\
\left.\text { Realschulbuchhandlung, I-II (Berlin, G. Reimer, } 1851^{2} ; 1886^{3}\right) .\end{array}$ \\
\hline Casaubonus & Casaubon I. (1604). \\
\hline $\begin{array}{l}\text { Forchhammer, } \\
\text { Topogr. Athenarum }\end{array}$ & $\begin{array}{l}\text { Forchhammer P.W., Müller K.O. (1833),Zur Topographie Athens. Ein Brief aus } \\
\text { Buchhandlung. ein Brief nach Athen, Göttingen, in der Dieterichschen }\end{array}$ \\
\hline $\begin{array}{l}\text { Forchhammer in } \\
\text { Alterthum. }\end{array}$ & $\begin{array}{l}\text { Forchhammer P.W. (1843), « Ueber die Stadtmauer Athens », Zeitschrift für die } \\
\text { Alterthumswissenschaft, I, 69, coll. 545-552-70, coll. 553-557. }\end{array}$ \\
\hline Geelius & $\begin{array}{l}\text { Geel J. (1840). } \\
\text { Görlitz J. (1832), "Dionis Chrysostomi de eloquentiae studio oratorio } \\
\text { Goerlitz } \\
\text { examendatius [...] ed ad audiendas iuvenum qui scholae valedicunt orationes [....], invitat } \\
\text { I.G., Vitenbergae, p. 1-11. }\end{array}$ \\
\hline $\begin{array}{l}\text { Goell. ad Argum. } \\
\text { Thuc. }\end{array}$ & $\begin{array}{l}\text { Göller F. (18362), Thucydidis de bello Peloponnesiaco libri octo, Vol. I, Libri I-IV, } \\
\text { Lipsiae, in Libraria Caroli Cnobloch. }\end{array}$ \\
\hline
\end{tabular}




\begin{tabular}{|c|c|}
\hline $\begin{array}{l}\text { Hemsterh. in } \\
\text { Anecdt. }\end{array}$ & $\begin{array}{l}\text { Geel J. (1825), Anecdota Hemsterhusiana, ex schedis mss., in bibliotheca Lugd. } \\
\text { Batava servatis, collegit, disposuit et edidit, Pars I, Lugduni Batavorum, apud S. et } \\
\text { J. Luchtmans. }\end{array}$ \\
\hline Jacobsius & $\begin{array}{l}\text { Jacobs F. (1834), «Spicilegium Annotationum in Orationes Dionis } \\
\text { Chrysostomi ", Zeitschrift für die Alterthumswissenschaft, I, 86, coll. 689-694. }\end{array}$ \\
\hline $\begin{array}{l}\text { Leake Topogr. Ath. } \\
\text { vers. germ. }\end{array}$ & $\begin{array}{l}\text { Leake W.M. (1829), Leake's Topographie von Athen, nebst einigen } \\
\text { Bemerkungen über die Alterthümer desselben, Aus dem Englischen übersetzt } \\
\text { und mit Anmerkungen von den Herren Professoren M.H.E. Meier und } \\
\text { K.O. Müller herausgegeben von A. Rienäcker, Halle, C.A. Kummel (2. } \\
\text { umgearb. Aufl. 1841). }\end{array}$ \\
\hline $\begin{array}{l}\text { Paroemiogr. Graec. } \\
\text { Leutsch. et } \\
\text { Schneidew. }\end{array}$ & $\begin{array}{l}\text { Leutsch E.L. von, Schneidewin F.G. (1839-1851), Corpus Paroemiographorum } \\
\text { Graecorum, Gottingae, I-II, Vandenhoeck \& Ruprecht (rist. Hildesheim, Olms, } \\
\text { 1958). }\end{array}$ \\
\hline Lobeck & $\begin{array}{l}\text { Lobeck Chr.A. (1835), Sophoclis Ajax, Commentario perpetuo illustravit. Editio } \\
\text { secunda novis curis elaborata, Berolini, apud Weidmannos (Lipsiae, in Libraria } \\
\text { Weidmannia, } 1809^{1} \text {; Berolini, apud Weidmannos, } 1866^{3} \text { ), p. 109-110. }\end{array}$ \\
\hline $\begin{array}{l}\text { Meinek. Fragm. } \\
\text { Men. }\end{array}$ & $\begin{array}{l}\text { Meineke A. (1823), Menandri et Philemonis reliquiae; accedunt R. Bentleii in } \\
\text { Menandrum et Philemonem emendationes integrae, Berolini, sumptibus Augustii } \\
\text { Mylii. }\end{array}$ \\
\hline Morellus & Morel F. (1604). \\
\hline $\begin{array}{l}\text { Mueller Comment. } \\
\text { de Munimentis } \\
\text { Athenarum }\end{array}$ & $\begin{array}{l}\text { Müller K.O. (1836), De munimentis Athenarum quaestiones historicae et tituli de } \\
\text { instauratione eorum perscripti explicatio. Commentationes duae, Gottingae, e } \\
\text { Libraria Dieterichiana. }\end{array}$ \\
\hline Poppo & $\begin{array}{l}\text { Poppo E.F. (1823), Thucydidis de bello Peloponnesiaco libri octo, Pars prima, } \\
\text { vol. II, Lipsiae, apud Gerhardum Fleischerum. }\end{array}$ \\
\hline Pflugkius & Pflugk J. (1831), p. 23. \\
\hline Reiskius & $\begin{array}{l}\text { Reiske J. (1784).[Selden J.] Notae et emendationes viri docti nescio cujus, ad } \\
\text { exemplum Dionis Chrysostomi edit. Paris. an. } 1623 \text { fol. adscriptae, quod exstat in } \\
\text { Biblioth. Bodleiana, in J.C. Wolf, Anecdota Graeca sacra et profana, [...], Tomus I, } \\
\text { Hamburgi, apud Theodorum Christophorum Felginer, 1722, p. 217-298. }\end{array}$ \\
\hline Vales. Anecdt. & $\begin{array}{l}\text { Valois H. de (1740), Emendationum libri quinque. Et de critica libri duo. Numquam } \\
\text { antehac typis vulgati. [...] Edente P.Burmanno, Amstelaedami, apud } \\
\text { Salomonem Schouten. }\end{array}$ \\
\hline Veneta & Dionis Chrysostomi orationes LXXX, Venetiis, Aldo Manuzio, s. d. [ca. 1550]. \\
\hline Westerm. Mythogr. & $\begin{array}{l}\text { Westermann А. (1843), МҮӨОГРАФОI. Scriptores poeticae historiae Graeci, } \\
\text { Brunsvigae. }\end{array}$ \\
\hline Wyttenb. (ad) Plut. & $\begin{array}{l}\text { Wyttenbach D. (1820-1821), Animadversiones in Plutarchi opera moralia ad } \\
\text { editionem Oxoniensem emendatius expressae [...], I-II, Oxonii, e Typographeo } \\
\text { Clarendoniano. }\end{array}$ \\
\hline
\end{tabular}

\section{NOTE}

1. Cf. Amato 2011, 140. 
2. Concepito come secondo volume della sua monumentale edizione di Dione Crisostomo (cf. Emper 1844, V), con il quale egli intendeva fornire un sussidio criticoesegetico, esso non poté vedere la luce a causa della morte prematura dell'autore così come del fallimento di ogni tentativo di pubblicazione postuma dell'autografo da parte degli amici e colleghi che se ne erano fatti carico. Per una puntuale ricostruzione delle vicende dell'autografo, costituito da ben $678 \mathrm{ff}$. ed entrato a far parte della biblioteca universitaria di Leida, dove è ancora conservato con la sigla $B P G 89$, si veda più diffusamente Amato 2011, 140-142.

3. Estratti si leggono in Amato 2011, 143-163, e in Ventrella 2012, 17-60.

4. Una nuova edizione critica del discorso è stata curata da Agus 1999 per la sua tèsi di dottorato, ma non mi è stato ancora possibile consultarla. L'edizione di Krapinger 1996 ripropone pedissequamente il testo greco dell'Arnim 1893.

5. Così Amato 2011, 142.

6. Riporto il testo greco quale da me stabilito, limitandomi ad indicare in apparato i dati relativi ai soli lemmi discussi. Con M si indica il Leid. B.P. gr. 2c, saec. XVI ; quanto ai nomi degli editori e studiosi abbreviati nell'apparato critico, essi vanno così sciolti : Cas. $=$ Casaubon $1604 ;$ Dind. $=$ Dindorf $1857 ;$ Emp. $=$ Emper $1844 ;$ Rei. $=$ Reiske 1784.

7. Cf. $\S 37,4$; § 55, 4 ; § 62, 4. Importante il parallelo indicato tra $\S 59,2$ e D.Chr. or. 3 , 116, parallelo la cui rilevanza ai fini della datazione del discorso dioneo è però sfuggita all'Emper ; sulla datazione dell'orazione, da ascrivere al periodo esilico, se non proprio a quello post-esilico della prima età traianea, cf. Ventrella 2015.

8. Cf. $\S 40,7$ e $\S 54,4$.

9. Significativo è il caso del $\S 10$, laddove contro la proposta di Pflugk 1831, 23, di

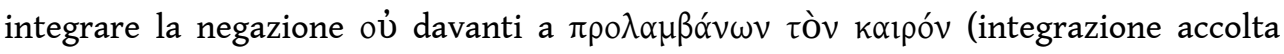
invece da Dindorf 1857, e passata nelle edizioni dell'Arnim 1893 e di Budé 1916), Emper manteneva saggiamente il testo tràdito. Analogamente al $\S 43,6$, l'editore rigetta

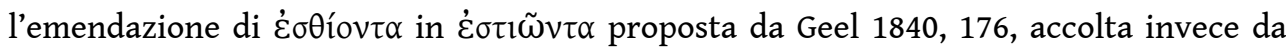
Dindorf 1857 e dall'Arnim 1893, ma poi respinta da G. de Budé.

10. Cf. $\S 50,9$; in $\S 34,12$ preferisce la lezione attestata pressoché unanimemente nei codici, con l'unica eccezione di C.

11. Cf. Reiske 1784, 199, n. 11.

12. Cf. Dukas 1810, 150.

13. Che Babilonia non disponesse di porti si desume da quanto raccontano Arriano (VII, 17, 5-6) e Diodoro Siculo (XVII, 116) della traversata dell'Eufrate da parte di Alessandro Magno.

14. Arriano (VII 21,1-3) ricorda i laghi siti lungo il canale Pallacotta (ad ottocento stadi da Babilonia), nei quali si faceva rifluire l'acqua dell'Eufrate ingrossato durante la stagione primaverile e presso al solstizio d'estate. Tra le opere idrauliche di Nitokris, Erodoto I, 185, 4, ricorda un lago artificiale che egli definisce come limne. Esso aveva dimensioni immense: milleduecento stadi per Ctesia (FGrHist $688 \mathrm{FI}$, 9), e 40 parasanghe per Abideno (fr. 9, 41-47 [FHG IV, p. 284]), secondo il quale il lago sarebbe stato invece opera di Nebuchadnezzar e sarebbe da localizzare presso Sippar; di varie limnai nei pressi di Babilonia si ricorda anche Appiano (Syr. 288).

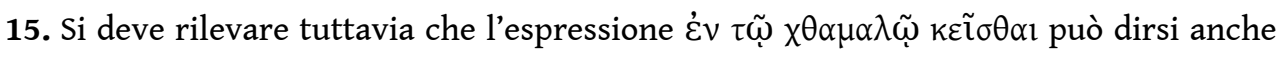

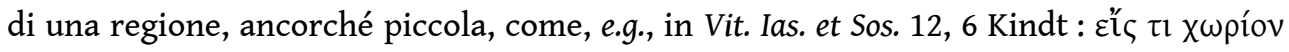




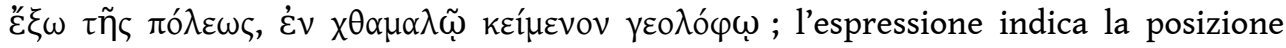
geografica di una città in Proc., De aed. 3, 4, 2.

16. Si veda, a tal proposito, Denniston 1950, 370 (i).

17. Per l'omissione di un sostantivo che si può dedurre dal contesto, si vedano Kühner \& Gerth $1898^{3}, 34, \S 352 \mathrm{~d}$; Boulenger 1922, 39-40; in particolare per l'ellissi del sostantivo $\pi$ ó $\lambda_{1 \varsigma}$, cf. Bos \& Schaefer 1825, p. 246-248.

18. Già Casaubon $1604,16-17$, proponeva di integrare oủ $\tilde{\eta} v$ prima di $\alpha$ Ủंòv, mentre

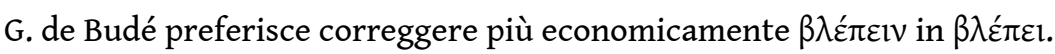

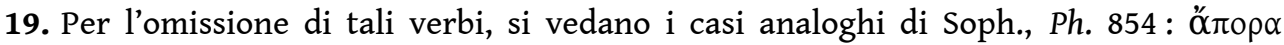

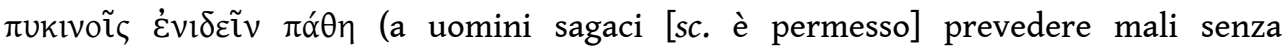
soluzione), dove il verbo $\dot{\varepsilon} v ı \delta \varepsilon \tilde{v}$ dipende da un « suppresso verbo, quod ipsa sententia

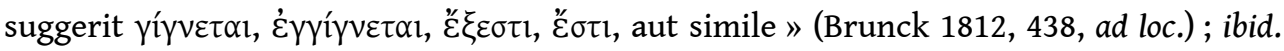

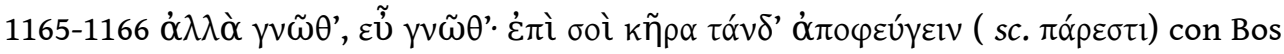
\& Schaefer $1825,381$.

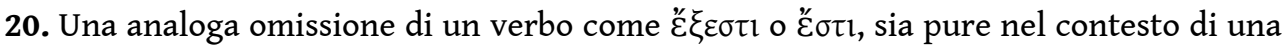

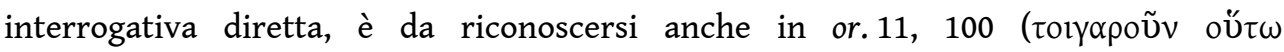

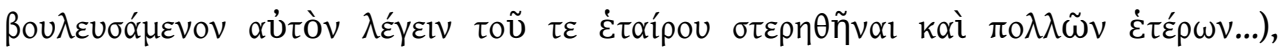

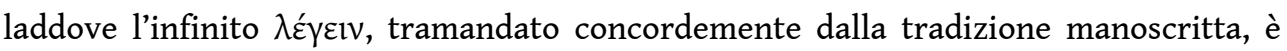
stato corretto (ingiustificatamente) in $\lambda \varepsilon^{\prime} \varepsilon \varepsilon$; sulla corretta interpretazione di tale passaggio (da intendersi nel senso di « è mai possibile dire che egli, presa tale decisione, perse... ?»), si veda ora Giangrande 2002, 96.

21. Per la trascrizione sono stati adottati gli stessi criteri indicati in Amato, Xenophontis ..., p. 142, n. 79, e Ventrella, Il commentario..., p. 17, n. 59. In altri termini, considerata la natura dello scritto, sono stati corretti tacitamente gli eventuali refusi, non si è dato conto delle abbondanti cancellature e aggiunte, così come dei frequenti spostamenti di parole presenti nell'autografo, né di eventuali ripensamenti da parte dell'autore, che, in taluni casi, pur avendo ripreso dal testo dioneo il lemma da commentare, ha poi omesso ogni tipo di esegesi. Le integrazioni apportate al testo sono segnalate dall'uso delle parentesi uncinate. Per una questione di uniformità e chiarezza tipografica, l'indicazione della pagina dell'inedito emperiano, sempre espressa in apice, è stata posta dopo il richiamo al § e alla linea del testo dioneo (secondo l'edizione curata dal medesimo studioso). I nomi Morellus, Reiskius, Geelius tra parentesi tonde stanno a indicare che L'Emper ha ripreso (più o meno verbatim) le osservazioni dei suoi predecessori. Per comodità del lettore, alla fine della trascrizione, abbiamo inserito una lista delle abbreviazioni delle opere citate, spesso in maniera piuttosto rapida, dall'Emper stesso. Nei casi in cui l'autore non ha indicato nel suo Commentario l'estensione esatta della citazione bibliografica o la stessa sia di difficile reperimento, si è preferito indicarla nella nostra lista. Un ringraziamento particolare va a Olivier Desbordes (Université de Caen Normandie) per la cura e l'attenzione con cui ha riletto il presente contributo, provvedendo a depurarlo dei refusi che ancora lo inquinavano. 


\section{RIASSUNTI}

L'articolo propone la prima trascrizione dal manoscritto Leid. BPG 89, ff. 94r-103r, contenente il commentario di A. Emper al $6^{\circ}$ discorso di Dione Crisostomo. In una breve introduzione all'inedito ci si propone di illustrare alcune delle più importanti novità introdotte dall'erudito con riguardo alla constitutio textus, così come di fornire nuove proposte critico-esegetiche.

The paper proposes the first transcription of the manuscript Leid. BPG 89, ff. 94r-103r, containing A. Emper's commentary on the 6th discourse of Dio Chrysostom. In a short introduction to the unpublished work we intend to illustrate some of the most important new points introduced by the scholar concerning the constitutio textus, as well as to provide new critical and exegetical suggestions.

\section{INDICE}

Parole chiave : Emper A., manoscritto Leid. BPG 89, Dione Crisostomo, Diogene o sulla tirannide (or. 6), note critico-esegetiche

Keywords : Emper A., manuscript Leid. BPG 89, Dio Chrysostom, Diogenes or On Tyranny (6th discourse), critical and exegetical notes

\section{AUTORE}

\section{GIANLUCA VENTRELLA}

EA 4276 L'Amo, Université de Nantes 